\title{
CRITICAL VALUES OF HOMOLOGY CLASSES OF LOOPS AND POSITIVE CURVATURE
}

\author{
HANS-BERT RADEMACHER
}

\begin{abstract}
We study compact and simply-connected Riemannian manifolds $(M, g)$ with positive sectional curvature $K \geq 1$. For a non-trivial homology class of lowest positive dimension in the space of loops based at a point $p \in M$ or in the free loop space one can define a critical length $\mathrm{crl}_{p}(M, g)$ resp. $\operatorname{crl}(M, g)$. Then $\mathrm{crl}_{p}(M, g)$ equals the length of a geodesic loop with base point $p$ and $\mathrm{crl}(M, g)$ equals the length of a closed geodesic. This is the idea of the proof of the existence of a closed geodesic of positive length presented by Birkhoff in case of a sphere and by Lusternik \& Fet in the general case. It is the main result of the paper that the numbers $\mathrm{crl}_{p}(M, g)$ resp. $\mathrm{crl}(M, g)$ attain its maximal value $2 \pi$ only for the round metric on the $n$-sphere.

Under the additional assumption $K \leq 4$ this result for $\operatorname{crl}(M, g)$ follows from results by Sugimoto in even dimensions and Ballmann, Thorbergsson \& Ziller in odd dimensions.
\end{abstract}

\section{Introduction}

For a compact Riemannian manifold $(M, g)$ let $\mathcal{M}=\{\gamma:[0,1] \longrightarrow$ $M ; \gamma$ absolutely continuous, $\left.\int_{0}^{1}\left\|\gamma^{\prime}\right\|^{2}(t) d t<\infty\right\}$ be the manifold of paths ( $H^{1}$-paths) on $M$. We consider in the sequel the following subspaces: the free loop space $\Lambda=\Lambda M=\{\gamma \in \mathcal{M} ; \gamma(0)=\gamma(1)\}$ and for $p, q \in M$ the space of paths between $p$ and $q: \Omega_{p q}=\Omega_{p q} M=\{\gamma \in \mathcal{M} ; \gamma(0)=p, \gamma(1)=q\}$. In particular for $p=q$ one obtains the (based) loop space $\Omega_{p} M=\Omega_{p p} M$, cf. [16, ch.2.3]. Now we use $X$ to denote one of the spaces $\Lambda M, \Omega_{p q} M, \Omega_{p} M$. We consider the energy functional $E: X \longrightarrow \mathbb{R}$ :

$$
E(\gamma)=\frac{1}{2} \int_{0}^{1}\left\|\gamma^{\prime}(t)\right\|^{2}(t) d t
$$

This is a differentiable function, the critical points are closed geodescis (if $X=\Lambda M$ ), geodesics joining $p$ and $q$ (if $X=\Omega_{p q} M$ ) and geodesic loops based at $p$ (if $X=\Omega_{p} M$ ).

For a critical point $c \in X$ we have $E(c)=L^{2}(c) / 2$, here $L(c)=\int_{0}^{1}\left\|c^{\prime}(t)\right\| d t$ is the length of $c$. We use the following notation for sublevel sets: $X \leq a:=$ $\{\gamma \in X ; E(\gamma) \leq a\}$ resp. $X^{<a}:=\{\gamma \in X ; E(\gamma)<a\}$ or $X^{a}:=\{\gamma \in X$; $E(\gamma)=a\}$.

Date: 2017-10-27.

2010 Mathematics Subject Classification. 53C20, 53C21, 53C22, 53C24, 58E10.

Key words and phrases. geodesic loops, loop space, free loop space, Morse theory, positive sectional curvature. 
The critical value (or critical level) $\operatorname{cr}(h)$ of a relative homology class $h \in$ $H_{k}\left(X, X^{\leq b}, \mathbb{Z}_{s}\right)$ is defined as follows:

(2)

$$
\operatorname{cr}(h)=\inf \left\{a \geq b ; h \in \operatorname{Image}\left(H_{k}\left(X^{\leq a}, X^{\leq b} ; \mathbb{Z}_{s}\right) \longrightarrow H_{k}\left(X, X^{\leq b} ; \mathbb{Z}_{s}\right)\right)\right\},
$$

cf. [15, ch.2.1] or [11, Sec.3]. Then $h \in H_{k}\left(X, X^{\leq b} ; \mathbb{Z}_{s}\right)$ is nontrivial if and only if $\operatorname{cr}(h)>b$. For a non-trivial class $h \in H_{k}\left(X ; \mathbb{Z}_{s}\right)$ there is a critical point $c \in X$ with $E(c)=\operatorname{cr}(h)$ resp. $L(c)=\sqrt{2 E(c)}$.

For a compact and simply-connected manifold $M=M^{n}$ of dimension $n$ let $k=k(M) \in\{1,2, \ldots, n-1\}$ be the unique number, such that $M$ is $k$ connected, but not $(k+1)$-connected. Hence we have the following statement about the homotopy groups: $\pi_{j}(M)=0$ for $1 \leq j \leq k$ and $\pi_{k+1}(M) \neq 0$. Then for $X=\Omega_{p q} M$ resp. $X=\Lambda M$ there is a smallest prime number $s \in \mathbb{P}$ such that $H_{k}\left(X, X^{0} ; \mathbb{Z}_{s}\right) \neq 0$. This follows since $\pi_{j}\left(\Omega_{p} M\right) \cong \pi_{j+1}(M)$ resp. $\pi_{j}(\Lambda M) \cong \pi_{j+1}(M) \oplus \pi_{j}(M)$, cf. [15, Cor. 2.1.5]. We define the critical length $\mathrm{crl}(M, g)$, resp. the critical length $\mathrm{crl}_{p}(M, g)$ at $p$ as follows:

$$
\operatorname{crl}(M, g):=\max \left\{\sqrt{2 \operatorname{cr}(h)} ; h \in H_{k(M)}\left(\Lambda M, \Lambda^{0} M ; \mathbb{Z}_{s}\right)\right\}
$$

and

$$
\operatorname{crl}_{p}(M, g):=\max \left\{\sqrt{2 \mathrm{cr}(h)} ; h \in H_{k(M)}\left(\Omega_{p} M ; \mathbb{Z}_{s}\right)\right\} .
$$

If the manifold $M$ is simply-connected and homeomorphic to a sphere $S^{n}$ then there is a uniquely determined generator $h \in H_{n-1}\left(\Lambda M, \Lambda^{0} M ; \mathbb{Z}_{2}\right) \cong$ $\mathbb{Z}_{2}$, resp. $h_{p} \in H_{n-1}\left(\Omega_{p} M ; \mathbb{Z}_{2}\right) \cong \mathbb{Z}_{2}$ such that $\operatorname{crl}(M, g)=\operatorname{cr}(h)$, resp. $\operatorname{crl}_{p}(M, g)=\operatorname{cr}\left(h_{p}\right)$. For a metric of positive sectional curvature we obtain the following result for the critical length resp. the critical length at a point. In particular we show that the maximal possible value is only attained for the round metric on a sphere of constant sectional curvature 1 :

Theorem 1. Let $\left(M^{n}, g\right)$ be a compact and simply-connected Riemannian manifold of positive sectional curvature $K \geq 1$. Then the critical lengths $\operatorname{crl}(M, g)$ and $\operatorname{crl}_{p}(M, g)$ for $p \in M$ satisfy:

(a) For all $p \in M: \operatorname{crl}_{p}(M, g) \leq 2 \pi$ and $\operatorname{crl}(M, g) \leq 2 \pi$.

(b) If for some $p \in M: \operatorname{crl}_{p}(M, g)>\pi$ or if $\operatorname{crl}(M, g)>\pi$ then the manifold is homeomorphic to the $n$-dimensional sphere $S^{n}$.

(b) If $\operatorname{crl}_{p}(M, g)=2 \pi$ for some $p \in M$, or if $\operatorname{crl}(M, g)=2 \pi$ then $(M, g)$ is isometric to the round sphere of constant sectional curvature 1.

Remark 1. (a) Note that the conclusions of the Theorem remain true if we replace $\operatorname{crl}(M, g)$ by $2 \operatorname{diam}(M, g)$, where $\operatorname{diam}(M, g)$ is the diameter of the Riemannian manifold. Then Part (a) is Bonnet's theorem, cf. [7, Thm.1.31], Part (b) is the generalized sphere theorem 9 by Grove \& Shiohama and Part (c) is Toponogov's maximal diameter theorem [7, Thm. 6.5], resp. 20. But one cannot reduce the proof of the Theorem to the statements for the diameter as examples constructed by Balacheff, Croke \& Katz [1] show: There is a one-parameter family $g_{t}, t \geq 0$ of smooth Zoll metrics on the 2-sphere such that the following holds: The metric $g_{0}$ is the round metric of constant curvature 1 and for $t>0$ the length $\mathcal{L}\left(S^{2}, g_{t}\right)$ of a shortest closed geodesic satisfies $\mathcal{L}\left(S^{2}, g_{t}\right)>2 \operatorname{diam}\left(S^{2}, g_{t}\right)$. 
(b) Since $\operatorname{crl}(M, g)>0$ one obtains the existence of a non-trivial closed geodesic of length $\mathrm{crl}(M, g)$, resp. a geodesic loop of length $\mathrm{crl}_{p}(M, g)$. This idea goes back to Birkhoff [5] in the case of a sphere and to Lyusternik-Fet [17] in the general case. Therefore we call $\mathrm{crl}(M, g)$ also the Birkhoff-length of the Riemannian manifold.

(c) Since for the number $k=k(M)$ we have $\pi_{k}\left(\Lambda M, \Lambda^{0} M\right) \cong \pi_{k}\left(\Omega_{p} M\right) \cong$ $\pi_{k+1}(M)$ and since for any $p \in M$ the based loop space $\Omega_{p} M$ can be seen as a subspace of the free loop space $\Lambda M$ we obtain for all $p \in M$ :

$$
\operatorname{crl}(M, g) \leq \operatorname{crl}_{p}(M, g) \text {. }
$$

In Example 1 we construct a one-parameter family $\left(S^{2}, g_{r}\right), 0<r \leq 1$ of convex surfaces of revolution with $\mathrm{crl}_{p}\left(S^{2}, g_{r}\right) \geq \pi$ for all $p \in S^{2}$, but $\lim _{r \rightarrow 0} \operatorname{crl}\left(S^{2}, g_{r}\right)=0$.

For a compact Riemannian manifold $(M, g)$ we denote by $\mathcal{L}=\mathcal{L}(M, g)$ the length of a shortest (non-trivial) closed geodesic. For a point $p \in M$ we denote by $\mathcal{L}(p)$ the length of a shortest (non-trivial) geodesic loop with initial point $p$. Then we have the obvious estimates: $\mathcal{L} \leq \operatorname{crl}(M, g)$ and $\mathcal{L}(p) \leq \operatorname{crl}_{p}(M, g)$. In particular we obtain as

Corollary 1. Let $\left(M^{n}, g\right)$ be a simply-connected Riemannian manifold of positive sectional curvature $K \geq 1$ and $p \in M$.

(a) $\mathcal{L} \leq 2 \pi$, and $\mathcal{L}(p) \leq 2 \pi$ for all $p \in M$.

(b) If $\mathcal{L}=2 \pi$ or if $\mathcal{L}(p)=2 \pi$ for some $p \in M$ then the manifold is isometric to the sphere of constant curvature 1 .

Toponogov proves in [21] that a simple closed geodesic (i.e. a closed geodesic with no self-intersection) on a convex surface with $K \geq 1$ has length $\leq 2 \pi$ with equality if and only if the sectional curvature is constant. He also shows the analogous statement for a geodesic loop. Statement (b) for $\mathcal{L}$ is claimed in several preprints (unpublished) by Itokawa \& Kobayashi [12], [13], [14]. In case of quaterly pinched metrics there are stronger results about a gap in the length spectrum obtained using the Toponogov triangle comparison results:

Theorem 2. Let $M$ be a manifold homeomorphic to $S^{n}$ carrying a Riemannian metric $g$ with sectional curvature $1 \leq K \leq \Delta \leq 4$.

(a) (Ballmann [2, Teil III]) The critical length $\mathrm{crl}(M, g)$ and the length $\mathcal{L}$ of a shortest closed geodesic coincide and satisfy: $2 \pi / \sqrt{\Delta} \leq \operatorname{crl}(M, g)=$ $\mathcal{L} \leq 2 \pi$

(b) (Ballmann,Thorbersson \& Ziller [4, Thm.1.7]) If there is a closed geodesic $c$ with length $2 \pi \leq L(c) \leq 4 \pi / \sqrt{\Delta}$ then the metric has constant sectional curvature.

Remark 2. (a) The case $n=2$ in Part (a) is also contained in 4, Thm. 4.2]. For even dimension Part (b) was shown by Sugimoto [19, Thm. B,C]. compare also the Remark following [4, Thm.1.7].

(b) In [2, Teil III] the following stronger statement is shown: For any shortest closed geodesic $c$ there is a homotopically non-trivial map $G:\left(D^{n-1}, \partial D^{n-1}\right) \longrightarrow\left(\{c\} \cup \Lambda^{<\mathcal{L}^{2} / 2} M, \Lambda^{<\mathcal{L}^{2} / 2} M\right)$. Hence the generator $h \in H_{n-1}\left(\Lambda M, \Lambda^{0} M ; \mathbb{Z}_{2}\right)$ remains hanging at any shortest closed geodesic. 
(c) In 3] the existence of $g(n)$ geometrically distinct closed geodesics on $S^{n}$ is shown under the assumptions of Theorem 2. The lengths of these closed geodesics lie in the interval $[2 \pi / \sqrt{\Delta}, 2 \pi]$ and $g(n)$ is the cup-length of the Grassmannian $G_{2}\left(\mathbb{R}^{n+1}\right)$ of unoriented 2-planes in $\mathbb{R}^{n+1}$. Instead of an assumption about the sectional curvature, which is an assumption depending continously on the metric and its first and second derivatives one can also investigate assumptions depending continuously only on the metric. For example Ballmann, Thorbergsson \& Ziller consider in [3, Sec.3] the so-called Morse-condition. Hingston shows in [10, Sec.5] a similar result about the existence of $g(n)$ closed geodesics. These results motivate Proposition 2. where we consider Riemannian metrics $g$ on $S^{n}$ satisfying $g \leq g_{1}$. Here $g_{1}$ is the round metric of constant curvature 1.

\section{RAUCH COMPARISON RESUlT}

Let $c: \mathbb{R} \longrightarrow M$ be a geodesic parametrized by arc length, then $c\left(t_{1}\right)$ is called focal point of $p=c(0)$ (along $c \mid\left[0, t_{1}\right]$ ) if there is a non-trivial normal Jacobi field $Y=Y(t)$ along $c$ with $\left\langle Y(t), c^{\prime}(t)\right\rangle=0$ for all $t$ and vanishing covariant derivative $\nabla Y(0) / d t=0$ (along the curve) and $Y\left(t_{1}\right)=0$, cf. [16, Def. 1.12.20]. The focal radius $\rho=\rho(M, g)$ of a compact Riemannian manifold $(M, g)$ equals the minimal positive number $t_{1}$ with the above property. The following statement is also called second Rauch comparison theorem (Rauch II), cf. [7, Thm.1.34], which is due to Berger. But note that we also include a rigidity statement, see for example [8, Thm.3.3].

Proposition 1. Let $c:[0, a] \longrightarrow M$ be a geodesic parametrized by arc length on a Riemannian manifold with positive sectional curvature $K \geq 1$ and let $c\left(t_{1}\right)$ be the first focal point. Let $Y=Y(t)$ be a Jacobi field along $c=c(t)$ with covariant derivative along $c: Y^{\prime}(0)=\nabla Y(0) / d t=0$. Then $\|Y(t)\| \leq\|Y(0)\| \cos (2 \pi t)$ for all $0 \leq t \leq t_{1}$. If $\left\|Y\left(t_{0}\right)\right\|=\|Y(0)\| \cos \left(2 \pi t_{0}\right)$ for some $t_{0} \in\left(0, t_{1}\right]$, then $\|Y(t)\|=\|Y(0)\| \cos (2 \pi t)$ for all $t \in\left[0, t_{0}\right]$ and $Y /\|Y\|$ is parallel along $c \mid\left[0, t_{0}\right]$.

Using this Rauch comparison result one obtains the following consequence. This result can be found for example in [7, Cor.1.36] or in [16, Cor. 2.7.10]. But note that we also include a rigidity statement. We denote by exp : $T M \longrightarrow M$ the exponential map, i.e. for $v \in T_{p} M$ the curve $t \in \mathbb{R} \longmapsto$ $\exp (t v)$ equals the geodesic $\gamma_{v}=\gamma_{v}(t)$ with initial point $p=\gamma_{v}(0)$ and direction $v=\gamma_{v}^{\prime}(0)$. And $\exp _{p}: T_{p} M \longrightarrow M$ denotes the restriction to the tangent space $T_{p} M$ at $p$.

Corollary 2. Let $c:[0, a] \longrightarrow M$ be a geodesic on a compact Riemannian manifold of positive sectional curvature $K \geq 1$ and focal radius $\rho>0$. Let $c_{1}:[0, a] \longrightarrow S_{1}^{n}$ be a great circle of length $L(c)$ on the standard sphere $S_{1}^{n}$ of constant sectional curvature 1. Choose parallel unit vector fields E along $c$ resp. $E_{1}$ along $c_{1}$ which are orthogonal to the geodesic $c$ resp. $c_{1}$. For a smooth non-negative function $f:[0, a] \longrightarrow \mathbb{R}$ with $0<f(t) \leq \rho$ for all $t \in(0, a)$ we define the smooth curve $b(t)=\exp _{c(t)}(f(t) E(t)) \in M, t \in[0, a]$ on $M$ resp. the curve $b_{1}(t)=\exp _{c_{1}(t)}\left(f(t) E_{1}(t)\right) \in S_{1}^{n}, t \in[0, a]$ on the round sphere $S_{1}^{n}$ of constant sectional curvature 1 . 
Then the following statements hold:

(a) $L(b) \leq L\left(b_{1}\right)$.

(b) Assume $L(b)=L\left(b_{1}\right)$. Let $f^{*}:[0, a] \longrightarrow \mathbb{R}$ be a non-negative function with $0<f^{*}(t) \leq f(t)$ for all $t \in(0, a)$ and define the curve $b^{*}(t)=$ $\exp _{c(t)}\left(f^{*}(t) E(t)\right) \in M, t \in[0, a]$ on $M$ resp. the following curve $b_{1}^{*}(t)=$ $\exp _{c_{1}(t)}\left(f^{*}(t) E_{1}(t)\right) \in S_{1}^{n}, t \in[0, a]$ on the round sphere $S_{1}^{n}$.

Then $L\left(b^{*}\right)=L\left(b_{1}^{*}\right)$.

Proof. The proof of (a) is given in [7, Cor. 1.36] or [16, Cor. 2.7.10].

Assume $L(b)=L\left(b_{1}\right)$. Let for $t \in[0, a]: s \in[0, f(t)] \longmapsto \gamma_{t}(s)=$ $\exp _{c(t)}(s E(t))$ denote the geodesic with $\gamma_{t}(0)=c(t)$ and $\gamma_{t}^{\prime}(0)=E(t)$ and let $s \in[0, f(t)] \longmapsto W_{t}(s)$ be the parallel unit vector field along $\gamma_{t}$ with $W_{t}(0)=$ $c^{\prime}(t)$ for all $t \in[0, a]$. We denote by $Y_{t}(s)=\partial \gamma_{t}(s) / \partial s$ the Jacobi field defined by the geodesic variation $t \longmapsto \gamma_{t}$, which satisfies $\left\|Y_{t}(f(t))\right\|=\cos (2 \pi f(t))$. It follows from the Proof of part (a) that $\left\|Y_{t}(s)\right\|=\cos (2 \pi s)$ for all $s \in[0, f(t)]$. Then we conclude from Proposition 1 that $W_{t}(s)=Y_{t}(s) / \cos (2 \pi s)$ is the parallel unit vector field along $c_{t}:[0, f(t)] \longrightarrow M$ with $W_{t}(0)=c^{\prime}(t)$. Then $U:=\left\{\exp _{c_{1}(t)}\left(s E_{1}(t)\right) \in S_{1}^{n} ; 0 \leq s \leq f(t)\right\}$ is a totally geodesic surface in the round sphere. Hence the map

$$
\exp _{c_{1}(t)}\left(s E_{1}(t)\right) \in U \subset S_{1}^{n} \longmapsto \exp _{c(t)}(s E(t)) \in M
$$

defines a totally geodesic isometric immersion of the totally geodesic surface $\left(U, g_{1}\right)$ in $S_{1}^{n}$ into the Riemannian manifold $(M, g)$. Therefore the claim follows.

\section{Morse Theory on Path Spaces}

The energy functional $E: \Omega_{p q} M \longrightarrow \mathbb{R}$ is differentiable and its critical points are geodesics $c \in \Omega_{p q} M$. The maximal dimension of a subspace of the tangent space $T_{c} \Omega_{p q} M$ on which the hessian $d^{2} E(c)$ is negative definite, is called index $\operatorname{ind}_{0}(c)$. If $p=q$ and if $c$ is a closed geodesic, then its index $\operatorname{ind}(c)$ as critical point of $E: \Lambda M \longrightarrow \mathbb{R}$ is analogously defined. In general the invariants $\operatorname{ind}_{0}(c)$ and ind $(c)$ differ, the difference $0 \leq \operatorname{conc}(c):=\operatorname{ind}(c)-$ $\operatorname{ind}_{0}(c) \leq n-1$ is called concavity, cf. [16, Thm.2.5.14]. The nullity null 0 (c) of a geodesic $c \in \Omega_{p q} M$ is defined as the dimension of the kernel of the hessian. It coincides with the dimension of Jacobi fields $Y=Y(t)$ along $c=c(t), t \in[0,1]$ which vanish at the end points, i.e. $Y(0)=Y(1)=0$. It follows that $0 \leq \operatorname{null}_{0}(c) \leq n-1$. If $c$ is a closed geodesic $c \in \Omega_{p} M \subset \Lambda M$ then the nullity null $(c)$ as critical point of the $S^{1}$-invariant energy functional $E: \Lambda M \longrightarrow \mathbb{R}$ equals the dimension of periodic and orthogonal Jacobi fields. Hence $0 \leq \operatorname{null}(c) \leq 2 n-2$.

The energy functional $E: \Omega_{p q} M \longrightarrow \mathbb{R}$ is a Morse function, if all geodesics $c \in \Omega_{p q} M$ are non-degenerate, i.e. $\operatorname{null}_{0}(c)=0$. This is the case if and only if $q \in M$ is a regular point of the exponential mapping $\exp _{p}: T_{p} M \longrightarrow M$, cf. [18, §18]. The Morse lemma implies that around any geodesic $c$ there are coordinates $(x, y) \in V_{-} \oplus V_{+}$with $E(x, y)=-\|x\|^{2}+\|y\|^{2}$. Here $V_{-}$ is a finite-dimensional subspace of dimension $\operatorname{ind}_{0}(c)$ on which the Hessian $d^{2} E(c)$ is negative definite and $V_{+}$is a closed complement in $T_{c} \Omega_{p q} M$. Let the energy functional $E: \Omega_{p q} M \longrightarrow \mathbb{R}$ be a Morse function, $l \in \mathbb{N}$ and let $a$ 
be a critical value such that for some $\epsilon>0$ the value $a$ is the only critical value in the interval $(a-2 \epsilon, a+2 \epsilon)$. Then the following holds: There are geodesics $c_{1}, \ldots, c_{r} \in \Omega_{p q} M$ with $E\left(c_{1}\right)=\ldots=E\left(c_{r}\right)=a$ and $\operatorname{ind}_{0}\left(c_{1}\right)=$ $\ldots=\operatorname{ind}_{0}\left(c_{r}\right)=l$ that for all $\delta \in(0, \epsilon]$ :

$$
H_{l}\left(\Omega_{p q}^{\leq a+\delta} M, \Omega_{p q}^{\leq a-\delta} M ; \mathbb{Z}_{2}\right) \cong \bigoplus_{k=1}^{r} \mathbb{Z}_{2}\left[F_{c_{k}}\right] .
$$

The relative homology class $\left[F_{c_{k}}\right] \in H_{l}\left(\Omega_{\bar{p} q}^{\leq a+\delta} M, \Omega_{\bar{p} q}^{\leq a-\delta} M ; \mathbb{Z}_{2}\right)$ can be represented by the set $\left\{(x, 0) ; x \in V_{-}\right\}$in a neigborhood of $c_{k}$ in which Morse coordinates $(x, y) \in V_{-} \oplus V_{+}$exist. The Morse Lemma implies that there is a sufficiently small neighborhood $U\left(c_{k}\right) \subset \Omega_{p q} M$ of $c_{k}$ such that the homology class $\left[F_{c_{k}}\right]$ is the unique generator of the critical group $H_{l}\left(\Omega_{\bar{p} q}^{\leq a-\delta} M \cup U\left(c_{k}\right)\right.$, $\left.\Omega_{p q}^{\leq a-\delta} M ; \mathbb{Z}_{2}\right) \cong \mathbb{Z}_{2}$

In the sequel we use an approximation argument. If $E: \Omega_{p} M \longrightarrow \mathbb{R}$ is not a Morse function, we choose a sequence $p_{j}$ of regular points for the exponential map $\exp _{p}$ converging to $p$. This is possible since the regular points of the exponential map are dense, cf. [18, Cor. 18.2].

\section{Critical values of homology Classes of loops}

Let $\operatorname{inj}=\operatorname{inj}(M, g)>0$ be the injectivity radius of the Riemannian manifold $(M, g)$. Then between two points $p, q$ of distance $d(p, q)<$ inj there is a unique minimal geodesic $c:[0,1] \longrightarrow M$ with $p=c(0), q=c(1)$ and $L(c)=d(p, q)$. Here $d(p, q)$ is the distance of the points $p$ and $q$.

Lemma 1. Let $p, q, r \in M$ with $d(q, r) \leq \min \{\operatorname{inj} / 2,1 / 2\}$ and let $c_{r q}$ : $[0, d] \longrightarrow M$ be the unique minimal geodesic joining $r=c_{r q}(0)=r, q=c_{r q}(1)$ with $L\left(c_{r q}\right)=d=d(q, r)$. Let $\zeta_{r q}: \Omega_{p q} M \longrightarrow \Omega_{p r} M$ be defined by

$$
\zeta_{r q}(\sigma)(t):=\left\{\begin{array}{cc}
\sigma(t /(1-d(q, r)) & ; \quad t \in[0,1-d(q, r)] ; \\
c_{r q}(t-(1-d(q, r))) & ; \quad t \in[1-d(q, r), 1]
\end{array} .\right.
$$

Let $h \in H_{j}\left(\Omega_{p q} M ; \mathbb{Z}_{s}\right)$ and let $\left(\zeta_{r q}\right)_{*}: H_{j}\left(\Omega_{p q} M ; \mathbb{Z}_{s}\right) \longrightarrow H_{j}\left(\Omega_{p r} M ; \mathbb{Z}_{s}\right)$ be the induced homomorphism. Then we obtain:

$$
(1-d(q, r)) \operatorname{cr}(h)-\frac{1}{2} d(q, r) \leq \operatorname{cr}\left(\left(\zeta_{r q}\right)_{*}(h)\right) \leq \frac{\operatorname{cr}(h)}{1-d(q, r)}+\frac{1}{2} d(q, r) .
$$

In particular the function $r \in M \longmapsto \operatorname{cr}\left(\left(\zeta_{r q}\right)_{*}(h)\right) \in \mathbb{R}^{+}$is continuous at $q$. Proof. For $\sigma:[0,1] \longrightarrow M$, with $p=\sigma(0), q=\sigma(1)$ and $r \in M$ with $d(q, r) \leq$ inj we obtain

$$
E\left(\zeta_{r q}(\sigma)\right)=\frac{E(\sigma)}{1-d(q, r)}+\frac{1}{2} d(q, r) .
$$

We conclude:

$$
\operatorname{cr}\left(\left(\zeta_{q r}\right)_{*}(h)\right) \leq \frac{\operatorname{cr}(h)}{1-d(q, r)}+\frac{d(q, r)}{2} .
$$

Applying this inequality to $\left(\zeta_{q r}\right)_{*}\left(\left(\zeta_{r q}\right)_{*}(h)\right)$ and using the fact that

$$
\operatorname{cr}(h)=\operatorname{cr}\left(\left(\zeta_{q r}\right)_{*} \circ\left(\zeta_{r q}\right)_{*}(h)\right)
$$


yields also the left inequality. Here we use that $\zeta_{q r} \circ \zeta_{r q}: \Omega_{p} M \longrightarrow \Omega_{p} M$ is homotopic to the identity map.

Lemma 2. Let $(M, g)$ be a compact Riemannian manifold of positive sectional curvature $K \geq 1$, and $p \in M:$ Let $h_{p} \in H_{k}\left(\Omega_{p} M ; \mathbb{Z}_{s}\right)$ be a non-zero homology class.

(a) If $k=n-1$ then $\sqrt{2 \mathrm{cr}\left(h_{p}\right)} \leq 2 \pi$.

(b) If $k \in\{1,2, \ldots, n-2\}$ then $\sqrt{2 \mathrm{cr}\left(h_{p}\right)} \leq \pi$.

Proof. Choose a sequence $p_{j}$ of regular points of $\exp _{p}$ with $\lim _{j \rightarrow \infty} d\left(p, p_{j}\right)=$ 0 , cf. for example [18, Cor. 18.2]. Then $E: \Omega_{p p_{j}} M \longrightarrow \mathbb{R}$ is a Morse function for any $j \geq 1$. Morse theory implies that there are finitely many geodesics $c_{j, 1}, \ldots, c_{j, r(j)} \in \Omega_{p p_{j}}(M)$ such that there exists one geodesic $c_{j, 1}$ with

$\sqrt{2 \mathrm{cr}\left(\left(\zeta_{p_{j} p}\right)_{*}\left(h_{p}\right)\right)}=L\left(c_{j, 1}\right)=\max \left\{L\left(c_{j, 1}\right), \ldots, L\left(c_{j, r(j)}\right)\right\}$ and $\operatorname{ind}_{0}\left(c_{j}\right)=$ $k$, cf. Section 3 .

(a) Since $K \geq 1$ and $k=n-1$ we conclude from Rauch comparison: $L\left(c_{j, 1}\right) \leq 2 \pi$, cf. [16, ch. 2.6]. Hence $\operatorname{cr}(h)=\lim _{j \rightarrow \infty} \operatorname{cr}\left(\left(f_{p_{j} p}\right)_{*}\left(h_{p}\right)\right) \leq 2 \pi^{2}$.

(b) Since $K \geq 1$ and $1 \leq k \leq n-2$ we conclude from Rauch comparison: $L\left(c_{j, 1}\right) \leq \pi$, cf. [16, ch. 2.6]. Hence $\operatorname{cr}(h)=\lim _{j \rightarrow \infty} \operatorname{cr}\left(\left(f_{p_{j} p}\right)_{*}\left(h_{p}\right)\right) \leq$ $\pi^{2} / 2$.

For a geodesic $c \in \Omega_{p q} M$ with $L(c)>\pi$ on a Riemannian manifold with $K \geq 1$ we construct a mapping $f_{c}: D^{n-1} \longrightarrow \Omega_{p q}^{\leq a} M$ which defines a relative homology class $\left[f_{c}\right] \in H_{n-1}\left(\Omega^{<E(c)} M \cup c, \Omega^{<E(c)} M ; \mathbb{Z}_{2}\right)$. If $E: \Omega_{p q} M \longrightarrow$ $\mathbb{R}$ is a Morse function, this relative homology class equals the homology class $\left[F_{c}\right]$ defined using the Morse Lemma, cf. Section 3 and Lemma 3 ,

This construction is motivated by a construction for symmetric spaces called cutting accross the corners for example in [6, Sec.10]. In case of the sphere $S^{n}$ and an arc $c:[0,1] \longrightarrow S^{n}$ of a great circle of length $\pi \leq L(c) \leq 2 \pi$ one starts with a cycle represented by the curves of length $L(c)$ formed by half-great circles through $p=c(0)$ and $-p=c(\pi / L(c))$ and the $\operatorname{arc} c(t), t \in$ $[\pi / L(c), 1]$. Hence all curves except $c$ are non-smooth geodesic polygons, which can be shortened by cutting accross the corners or using the negative gradient flow of $E$.

Definition 1. Let $(M, g)$ be a compact and simply-connected Riemannian manifold of positive sectional curvature $K \geq 1$ and focal radius $\rho$. Let $c$ : $[0,1] \longrightarrow M$ be a geodesic joining $p=c(0)$ and $q=c(1)$ with length $L(c) \geq \pi$. We define a mapping

$$
f_{c}: D^{n-1}(\rho) \longrightarrow \Omega_{p q} M
$$

as follows: We identify the $(n-1)$-dimensional disc $D^{n-1}(\rho)=\left\{x \in \mathbb{R}^{n-1}\right.$; $\left.\|x\|^{2} \leq \rho\right\}$ with the set $D^{n-1}(\rho)=\left\{w \in T_{p} M ;\left\langle w, c^{\prime}(0)\right\rangle=0,\|w\| \leq \rho\right\}$ of tangent vectors at $p$ orthogonal to $c^{\prime}(0)$ with norm $\leq \rho$. Let $\widetilde{f}_{c}: D^{n-1}(\rho) \longrightarrow$ $\Omega_{p q} M$ be defined by

$$
\widetilde{f}_{c}(v)(t)=\left\{\begin{array}{cl}
\exp _{c(t)}\left(g_{v}(t) V_{v}(t)\right) & ; 0 \leq t \leq \pi / L(c) \\
c(t) & ; \pi / L(c) \leq t \leq 1
\end{array} .\right.
$$

Here $V_{v}=V_{v}(t)$ is the parallel unit vector field along c with $V_{v}(0)=v /\|v\|, v \in$ $D^{n-1}(\rho)$. The function $g_{v}:[0,1] \longrightarrow(-\pi / 2, \pi / 2), v \in D^{n-1}(\rho)$ is the 
uniquely determined smooth function with $g_{v}(0)=0$ and $g_{v}(\pi /(2 L(c)))=$ $\|v\|$ such that the following holds: For the standard round metric on $S^{n}$ let $\widetilde{\phi}_{c}: D^{n-1}(\pi / 2) \longrightarrow \Omega_{p q} S^{n}$ be the map $\widetilde{f}_{c}$ introduced above in Equation (10). Then for a parametrization $c:[0,1] \longrightarrow S^{n}$ of a part of a great circle on the round sphere $S^{n}$ proportional to arc length with $L(c) \geq \pi$ the curves $t \in[0, \pi / L(c)] \longmapsto \widetilde{\phi}_{c}(v)(t) \in S^{n}$ are half great circles joining $p$ and $-p=c(\pi / L(c))$ with $\widetilde{\phi}_{c}(v)(\pi /(2 L(c)))=\exp _{c(\pi /(2 L(c)))}\left(\|v\| V_{v}(\pi /(2 L(c)))\right)$ up to parametrization.

Let $t \in[0,1] \longrightarrow \bar{f}_{c}(v)(t) \in M$ be the reparametrization of $t \in[0,1] \longrightarrow$ $\tilde{f}_{c}(v)(t) \in M$ such that the following holds: $\bar{f}_{c}(v)(t)=c(t)$ for all $\pi / L(c) \leq$ $t \leq 1$ and $t \in[0, L(c) / \pi] \longmapsto \bar{f}_{c}(v)(t) \in M$ is parametrized proportional to arc length. Denote by $\Phi^{s}: \Omega_{p q} M \longrightarrow \Omega_{p q} M, s>0$ the negative gradient flow of E. I.e.

$$
\left.\frac{d \Phi^{s}(\sigma)}{d s}\right|_{s=t}=-\operatorname{grad} E\left(\Phi^{s}(\sigma)\right) .
$$

Then finally $f_{c}(v):=\Phi^{1}\left(\bar{f}_{c}(v)\right)$ and $\phi_{c}(v):=\Phi^{1}\left(\bar{\phi}_{c}(v)\right)$

Remark 3. The formulae from spherical trigonometry imply:

$$
g_{v}(t)=\arctan (\sin (L(c) t) \tan \|v\|) .
$$

It is an observation by Itokawa \& Kobayashi [14, p.10-11] that using a consequence of a Corollary from the so-called Rauch's second comparison result, cf. Section 3 one can compare the lengths $L\left(\bar{f}_{c}(v)\right)$ and $L\left(\bar{\phi}_{c}(v)\right)$. Compare also Corollary 2 which also deals with the rigidity case.

Lemma 3. Let $(M, g)$ be a compact and simply-connected Riemannian manifold of positive sectional curvature $K \geq 1$ and focal radius $\rho$. Let $c:[0,1] \longrightarrow$ $M$ be a geodesic joining $p=c(0)$ and $q=c(1)$ with length $L(c) \geq \pi$, i.e. $E(c) \geq \pi^{2} / 2$. Let $f_{c}: D^{n-1}(\rho) \longrightarrow \Omega_{p q} M$ be the mapping introduced in Definition 1 .

(a) The mapping $f_{c}$ satisfies the following property: For all $v \in D^{n-1}(\rho)$ :

$$
E\left(f_{c}(v)\right) \leq E\left(f_{c}(0)\right)=E(c) .
$$

If $L(c)>\pi$ then there exists $\epsilon>0$, such that

$$
E\left(f_{c}(v)\right)<E\left(f_{c}(0)\right)=E(c)
$$

for all $v \in D^{n-1}(\rho), v \neq 0$ and

$$
E\left(f_{c}(v)\right) \leq E(c)-\epsilon
$$

for all $v \in \partial D^{n-1}(\rho)=\left\{v \in D^{n-1}(\rho) ;\|v\|=\rho\right.$. $\}$. Hence $f_{c}$ defines a relative homology class:

$$
\left[f_{c}\right] \in H_{n-1}\left(\Omega_{p q} M, \Omega_{p q}^{\leq E(c)-\epsilon} M ; \mathbb{Z}_{2}\right) .
$$

(b) If $L(c) \in(\pi, 2 \pi)$ and if $q$ is a regular point for $\exp _{p}$ and if $\operatorname{ind}_{0}(c)=$ $n-1$ then there is an $\epsilon>0$ such that for all $\delta \in(0, \epsilon]$ :

$$
0 \neq\left[f_{c}\right] \in H_{n-1}\left(\Omega_{p q}^{\leq E(c)+\delta} M, \Omega_{p q}^{\leq E(c)-\delta} M ; \mathbb{Z}_{2}\right) .
$$

(c) Let $q$ be a regular point for $\exp _{p}$ and let $0 \neq h \in H_{n-1}\left(\Omega_{p q} M ; \mathbb{Z}_{2}\right)$ with critical value $a:=\operatorname{cr}(h) \in\left(\pi^{2} / 2,2 \pi^{2}\right]$. Then there is a geodesic $c \in \Omega_{p q} M$ 
with $E(c)=a, \operatorname{ind}_{0}(c)=n-1$ and an $\epsilon>0$ such that the mapping $f_{c}$ defines a non-trivial relative homology class

$$
0 \neq\left[f_{c}\right] \in H_{n-1}\left(\Omega_{p q} M, \Omega_{p q}^{\leq a-\delta} M ; \mathbb{Z}_{2}\right)
$$

for all $\delta \in(0, \epsilon]$.

Proof. (a) We denote by $f_{c}, \widetilde{f}_{c}, \bar{f}_{c}: D^{n-1}(\rho) \longrightarrow \Omega_{p q} M$ the mappings introduced in Definition 1 for the Riemannian manifold $(M, g)$ and by $\phi_{c}, \widetilde{\phi}_{c}, \bar{\phi}_{c}$ : $D^{n-1}(\pi / 2) \longrightarrow \Omega_{p q} S^{n}$ the mappings associated to the standard round metric of constant sectional curvature 1 on the $n$-sphere $S^{n}$. Hence $t \in[0, \pi / L(c)] \longmapsto$ $\widetilde{\phi}_{c}(v)(t) \in S^{n}$ is a parametrization of a half great circle and $t \in[0, \pi / L(c)] \longmapsto$ $\bar{\phi}_{c}(v)(t) \in S^{n}$ is its parametrization proportional to arc length. Hence $L\left(\widetilde{\phi}_{c}(v)\right)=L\left(\bar{\phi}_{c}(v)\right)=L(c)$. Then Corollary 2 implies:

$$
\begin{array}{r}
L\left(\widetilde{f}_{c}(v) \mid[0, \pi / L(c)]\right)=L\left(\bar{f}_{c}(v) \mid[0, \pi / L(c)]\right) \\
\leq L\left(\widetilde{\phi}_{c}(v) \mid[0, \pi / L(c)]\right)=L\left(\bar{\phi}_{c}(v) \mid[0, \pi / L(c)]\right)=\pi
\end{array}
$$

since $K \geq 1$. This shows Equation (12). Now assume $L(c) \in(\pi, 2 \pi]$. Then for $v \neq 0$ the curves $\bar{f}_{c}(v)$ are not smooth at $t_{1}=\pi / L(c)$, hence

$$
E\left(\Phi^{1}\left(\bar{f}_{c}(v)\right)\right)<E\left(\bar{f}_{c}(v)\right) \leq E(c) .
$$

This proves Equation (13) and Equation (15).

(b) We define homotopies $f_{c, u}, \widetilde{f}_{c, u}, \bar{f}_{c, u}: D^{n-1}(\rho) \longrightarrow \Omega_{p q} M$ with $u \geq$ $\pi / L(c)$ of the mappings $f_{c}=f_{c, \pi / L(c)}, \widetilde{f}_{c}=\widetilde{f}_{c, \pi / L(c)}, \bar{f}_{c}=\bar{f}_{c, \pi / L(c)}$ resp. homotopies $\phi_{c, u}, \widetilde{\phi}_{c, u}, \bar{\phi}_{c, u}: D^{n-1}(\rho) \longrightarrow \Omega_{p q} M$ with $u \geq \pi / L(c)$ of the mappings $\phi_{c}=\phi_{c, \pi / L(c)}, \widetilde{\phi}_{c}=\widetilde{\phi}_{c, \pi / L(c)}, \bar{\phi}_{c}=\bar{f}_{c, \pi / L(c)}$ as follows: For $v \in$ $D^{n-1}(\rho), u \in(\pi / L(c), 2 \pi / L(c))$ let

$$
g_{v, u}(t)=\arctan \left(\sin (L(c) t) \frac{\tan \|v\|}{\sin (L(c) u / 2)}\right) \text {. }
$$

Let

$$
\widetilde{f}_{c, u}(t)=\left\{\begin{array}{cc}
\exp _{c(t)}\left(g_{v, u}(t) V_{v}(t)\right) & ; \quad 0 \leq t \leq u / 2 \\
\exp _{c(t)}\left(g_{v, u}(u-t) V_{v}(t)\right) & ; \quad u / 2 \leq t \leq u \\
c(t) & ; \quad u \leq t \leq 1
\end{array}\right.
$$

Let $t \in[0, u] \longrightarrow \bar{f}_{c, u}(v)(t)$ be the parametrization of $t \in[0, u] \longrightarrow \widetilde{f}_{c, u}(v)(t)$ proportional to arc length. We denote by $\bar{\phi}_{c, u}(v), \widetilde{\phi}_{c, u}(v)$ the corresponding mappings for the standard round metric on $S^{n}$. Using the formulae from spherical trigonometry one can check that the function $g_{v, u}(t)$ is the uniquely defined smooth function such that $t \in[0, u] \longmapsto \widetilde{\phi}_{c, u}(v)(t)=$ $=\exp _{c(t)}\left(g_{v, u}(t) E_{v}(t)\right) \in S^{n}$ is a parametrization of the arc of a great circle joining $p=c(0)$ and $\exp _{c(u / 2)}\left(\|v\| V_{v}(u / 2)\right)$. From spherical trigonometry we conclude for the length $L(u, v)=L\left(\bar{\phi}_{c, u}(v) \mid[0, u / 2]\right)=L\left(\bar{\phi}_{c, u}(v) \mid[u / 2, u]\right)$ of the great circle arcs:

$$
L(u, v)=\arccos \left(\cos \left(L(c) \frac{u}{2}\right) \cos \|v\|\right) .
$$


We compute:

$$
\left.\frac{\partial^{2} L\left(\bar{\phi}_{c, u}(v)\right)}{\partial v_{i}^{2}}\right|_{v_{i}=0}=\frac{\cos (L(c) u / 2)}{\sqrt{1-\cos ^{2}(L(c) u / 2)}}
$$

We conclude that for $u>\pi / L(c)$ the Hessian $d^{2}\left(E \circ \bar{\phi}_{c, u}\right)(0)$ at the point $0 \in D^{n-1}(\pi / 2)$ is negative definite, i.e. $c=\bar{\phi}_{c, u}(0)$ is a non-degenerate maximum point of the restriction $E: \bar{\phi}_{c, u}\left(D^{n-1}(\pi / 2)\right) \longrightarrow \mathbb{R}$. Using again Corollary 2 we obtain, that $E\left(\bar{f}_{c, u}(v)\right) \leq E\left(\bar{\phi}_{c, u}(v)\right)$ for all $u \in(\pi / L(c), 2 \pi / L(c))$, $v \in D^{n-1}(\rho)$. Therefore $c=\bar{f}_{c, u}(0)$ is also a non-degenerate maximum point of the restriction of the energy functional $E: \bar{f}_{c, u}\left(D^{n-1}(\rho)\right) \longrightarrow$ $\mathbb{R}$ to the local $(n-1)$-dimensional submanifold $\bar{f}_{c, u}\left(D^{n-1}(\rho)\right)$. Consider $\Phi^{1} \circ \bar{f}_{c, u}: D^{n-1}(\rho) \longrightarrow \Omega_{p q}^{\leq E(c)} M, u \geq \pi / L(c)$. Then there is $\epsilon>0$ such that $\Phi^{1}\left(\bar{f}_{c, u}\left(\partial D^{n-1}(\rho)\right)\right) \subset \Omega_{p q}^{\leq E(c)-\epsilon} M$ for all $u \geq \pi / L(c)$.

This shows that $f_{c, u}$ defines a non-zero homology class

$$
0 \neq\left[f_{c, u}\right] \in H_{n-1}\left(\Omega_{p q}^{\leq E(c)+\delta} M, \Omega_{p q}^{\leq E(c)-\delta} M ; \mathbb{Z}_{2}\right)
$$

for all $\delta \in(0, \epsilon]$ and $u>\pi / L(c)$. This holds by the Morse-Lemma since $c$ is a non-degenerate critical point of $E: \Omega_{p q}^{\leq E(c)+\epsilon} M \longrightarrow \mathbb{R}$ with $\operatorname{ind}_{0} c=n-1$, cf. Section 3, Define

$$
f_{c, u}:=\Phi^{1}\left(\bar{f}_{c, u}\right):\left(D^{n-1}(\rho), \partial D^{n-1}(\rho)\right) \longrightarrow\left(\Omega_{p q}^{\leq E(c)+\delta} M, \Omega_{p q}^{\leq E(c)-\delta} M\right) .
$$

for $u \geq \pi / L(c)$. The clue here is that by introducing the flow $\Phi^{1}$ this homotopy is also defined for $u=\pi / L(c)$. Then Equation (19) implies:

$$
0 \neq\left[f_{c}\right]=\left[f_{c, u}\right] \in H_{n-1}\left(\Omega_{p q}^{\leq E(c)+\delta} M, \Omega_{p q}^{\leq E(c)-\delta} M ; \mathbb{Z}_{2}\right)
$$

for some $u>\pi / L(c)$.

(c) We conclude from Part (b) and Section 3, There are finitely many geodesics $c_{1}, \ldots, c_{r} \in \Omega_{p q} M$ with $\operatorname{ind}_{0}\left(c_{1}\right)=\ldots=\operatorname{ind}_{0}\left(c_{r}\right)=n-1$ and $E\left(c_{1}\right)=E\left(c_{2}\right)=\ldots=E\left(c_{r}\right)=a$ such that the class $h$ as relative class in $H_{n-1}\left(\Omega_{\bar{p} q}^{\leq a+\delta} M, \Omega_{\bar{p} q}^{\leq a-\delta} M ; \mathbb{Z}_{2}\right)$ for some $\delta>0$ has the following representation: For sufficiently small $\epsilon>0$ the mappings $f_{c_{j}}:\left(D^{n-1}(\rho), \partial D^{n-1}(\rho)\right) \longrightarrow$ $\left(\Omega_{\bar{p} q}^{\leq a+\delta} M, \Omega_{\bar{p} q}^{\leq a-\delta} M\right), j=1,2, \ldots, r$ represent non-trivial relative homology classes $0 \neq\left[f_{c_{j}}\right]=\left[F_{c_{j}}\right] \in H_{n-1}\left(\Omega_{\bar{p} q}^{\leq a+\epsilon} M, \quad \Omega_{\bar{p} q}^{\leq a-\epsilon} M ; \mathbb{Z}_{2}\right), j=1,2, \ldots, r$ for all $\delta \in(0, \epsilon]$. Hence in $H_{n-1}\left(\Omega_{\bar{p} q}^{\leq a+\delta} M, \Omega_{\bar{p} q}^{\leq a-\delta} M ; \mathbb{Z}_{2}\right)$ we have the following representation: $h=\left[f_{c_{1}}\right]+\ldots+\left[f_{c_{r}}\right]$. Hence the image of $h$ under the homomorphism

$$
H_{n-1}\left(\Omega_{p q}^{\leq a+\delta} M ; \mathbb{Z}_{2}\right) \longrightarrow H_{n-1}\left(\Omega_{p q} M, \Omega_{p q}^{\leq a-\delta} M ; \mathbb{Z}_{2}\right)
$$

is mapped onto $\alpha_{*}\left(\sum_{j=1}^{r}\left[f_{c_{j}}\right]\right)$. Here

$$
\alpha_{*}: H_{n-1}\left(\Omega_{p q}^{\leq a+\delta} M, \Omega_{p q}^{\leq a-\delta} M ; \mathbb{Z}_{2}\right) \longrightarrow H_{n-1}\left(\Omega_{p q} M, \Omega_{p q}^{\leq a-\delta} M ; \mathbb{Z}_{2}\right)
$$


is the homomorphism induced by the inclusion. If $\alpha_{*}\left[f_{c_{j}}\right]=0$ for all $j=$ $1,2, \ldots, r$ then $h$ lies in the image of the homomorphism

$$
H_{n-1}\left(\Omega_{p q}^{\leq a-\delta} M ; \mathbb{Z}_{2}\right) \longrightarrow H_{n-1}\left(\Omega_{p q} M ; \mathbb{Z}_{2}\right),
$$

i.e. $\operatorname{cr}(h) \leq a-\delta$. This is a contradiction.

Lemma 4. Let $(M, g)$ be a compact and simply-connected Riemannian manifold of positive sectional curvature $K \geq 1$ :

(a) Let $\gamma \in \Omega_{q p} M$ be a geodesic of length $L(\gamma)=\pi$ joining $q=\gamma(0)$ and $p=\gamma(1)$. If $\gamma$ is not minimal, i.e. if $d(p, q)<\pi$ then there is a continuous path $s \in[0,1] \longmapsto \gamma_{s} \in \Omega_{q p} M$ with $\gamma_{0}=\gamma, E\left(\gamma_{s}\right) \leq \pi^{2} / 2$ for $s \in(0,1)$ and $E\left(\gamma_{1}\right)<\pi^{2} / 2$. If $E\left(\gamma_{s_{1}}\right)=\pi^{2} / 2$, then $E\left(\gamma_{s}\right)=\pi^{2} / 2$ for all $s \in\left[0, s_{1}\right]$.

(b) If $c \in \Omega_{p} M$ is a geodesic loop with $p=c(0), q=c(1 / 2)$ and length $L(c)=2 \pi$ then there is a path $s \in[0,1] \longmapsto c_{s} \in \Omega_{\bar{p}}^{\leq 2 \pi^{2}} M$ such that the following holds: For $s \in[0,1 / 2]$ the curves $c_{s}$ are geodesics with $L\left(c_{s}\right)=$ $2 \pi, c_{s}(1 / 2)=q$. And $E\left(c_{s}\right)<2 \pi^{2}$ for all $s \in(1 / 2,1]$.

Proof. (a) Define the subset

$$
A:=\left\{y \in T_{q} M ;\|y\|=\pi, \exp _{q}(y)=p\right\} .
$$

of the set $T_{q}^{\pi} M:=\left\{y \in T_{q} M ;\|y\|=\pi,\right\}$ of tangent vectors at $q$ of norm $\pi$. For $y \in A$ denote by

$$
c_{y}:[0,1] \longrightarrow M, c_{y}(t)=\exp _{q}(t y)
$$

the geodesic of length $\pi$ joining $q=c_{y}(0)$ and $p=c_{y}(1)=\exp _{q}(y)$. For $y \in A$ one can consider the mapping $f_{c_{y}}: D^{n-1}(\rho) \longrightarrow \Omega_{q p}^{\leq \pi^{2} / 2} M$ defined in Definition 1. compare Lemma 3 resp. Equation (12). For $y \in A$ define

$$
\eta_{y}:=\sup \left\{\eta \in[0, \rho] ; E\left(f_{c_{y}}\left(D^{n-1}(\eta)\right)\right)=\kappa^{2} / 2\right\} .
$$

If $\eta_{y}>0$ for $y \in A$ then the curves $t \in[0,1] \longmapsto f_{c_{y}}(v)(t) \in M$ with $\|v\| \leq \eta_{y}$ have energy $\pi^{2} / 2$ and are therefore by construction of $f_{c_{y}}$ geodesics. This implies that there is a neigborhood $U$ of $y$ in $A$ such that for $z \in U: \eta_{z}>0$. Hence $y$ is a point in the interior $\AA$ of the set $A$. By assumption $c^{\prime}(0) \in A$, let $B \subset A$ be the path-component of $A$ containing $c^{\prime}(0)$. $B$ is a closed subset of $T_{q}^{\pi} M=\left\{v \in T_{q} M ;\|v\|=\pi\right\}$. Now assume that for all $y \in B: \eta_{y}>0$. Then the set $B \subset T_{q}^{\pi} M$ is also open, i.e. $B=T_{q}^{\pi} M$. Hence $d(q, p)=\pi$ in contradiction to the assumption. Therefore there is $y \in B$ with $\eta_{y}=0$. Then one can construct a path $s \in[0,1] \longrightarrow \Omega_{q p} M \leq \pi^{2} / 2 M$ with the following properties: The restriction $s \in[0,1 / 2] \mapsto \alpha_{s} \in \Omega_{q p} M$ is a path consisting of geodesics of length $\pi$ joining $\alpha_{0}=c$ and $\alpha_{1 / 2}=c_{y}$, i.e. $\alpha_{s}^{\prime}(0) \in B$. And $s \in$ $[1 / 2,1] \mapsto \alpha_{s}(v)=f_{c_{y}}(v(s)) \in \Omega_{q p}^{\leq \pi^{2} / 2} M$ is a path satisfying $E\left(\alpha_{s}\right)<\pi^{2} / 2$ for all $s \in(1 / 2,1]$ and a path $s \in[1 / 2,1] \mapsto v(s) \in D^{n-1}(\rho)$. This is possible since $\eta_{y}=0$ and by the equality discussion in Corollary 2

(b) Let $\delta: T_{q}^{\pi} M \times T_{q}^{\pi} M \longrightarrow \mathbb{R}$ be the distance induced by the Riemannian metric $g_{q} / \pi^{2}$ on the sphere $T_{q}^{\pi} M$. This is the standard round metric of sectional curvature 1 on $T_{q}^{\pi} M$. We define the numbers

$$
\theta_{ \pm}:=\sup \left\{\theta \geq 0 ; \forall w \in T_{q}^{\pi} M, \delta\left( \pm c^{\prime}(1 / 2), w\right) \leq \theta: \exp _{q}(w)=p\right\} .
$$


Then it follows that there are $y_{ \pm} \in T_{q}^{\pi} M$ with $\delta\left( \pm c^{\prime}(1 / 2), y_{ \pm}\right)=\theta_{ \pm}$and $\eta_{y_{ \pm}}=0$. Then $0 \leq \theta_{ \pm}<\pi$ since $d(p, q)<\pi$. Without restriction we can assume that $\theta_{-} \geq \theta_{+}$. Then there exists a geodesic $\widetilde{c} \in \Omega_{p} M$ with $\delta\left(c^{\prime}(1 / 2), \widetilde{c}(1 / 2)=\theta_{+}\right.$and $\eta\left(\widetilde{c}^{\prime}(1 / 2)=0\right.$. Then $s \in[0,1 / 2] \mapsto c_{s} \in \Omega_{p}^{2 \pi^{2}} M$ is a path of geodesics of length $2 \pi$ joining $c_{0}=c$. In addition $c_{1 / 2}=\widetilde{c}$ and $c_{s} \in \Omega_{p} M$ for $s \in[1 / 2,1]$ is a path with $c_{s}(t)=\widetilde{c}(t)$ for $t \in[0,1 / 2]$ and $\gamma_{s}=c_{s} \mid[1 / 2,1]$ is a curve with $E\left(\gamma_{s}\right)<\pi^{2} / 2$ for all $s \in(1 / 2,1]$ as constructed in part (a).

Lemma 5. Let $(M, g)$ be a compact and simply-connected Riemannian manifold with positive sectional curvature $K \geq 1$ and let $c \in \Omega_{p} M$ be a geodesic loop based at $p$ of length $2 \pi$, resp. energy $2 \pi^{2}$. For sufficiently small positive $\epsilon>0$ the mapping $f_{c}: D^{n-1}(\rho) \longrightarrow \Omega_{p} M$ introduced in Definition 1 with $E\left(f_{c}(v)\right)<2 \pi^{2}$ for all $v \neq 0$ defines a relative homology class

$$
\left[f_{c}\right] \in H_{n-1}\left(\Omega_{p} M, \Omega_{p}^{\leq 2 \pi-\epsilon} M ; \mathbb{Z}_{2}\right) .
$$

If its critical value satisfies $\mathrm{cr}\left(\left[f_{c}\right]\right)=2 \pi$ then the Riemannian manifold is a round sphere of constant sectional curvature 1.

Proof. If the sectional curvature is not constant, the diameter $\operatorname{diam}(M, g)$ satisfies $\operatorname{diam} M<\pi$. This is a consequence of Toponogov's maximal diameter theorem [7, Thm. 6.5] resp. [20].

Let $q=c(1 / 2), p=c(0)=c(1)$. Then $d(q, p) \leq \operatorname{diam}(M, g)<\pi$. We conclude from Lemma 4 that there is a path $s \in[0,1] \longmapsto c_{s} \in \Omega_{p} M$ with $c_{0}=c, c_{1 / 2}=\widetilde{c} \in \Omega_{p} M$ a geodesic with $L(\widetilde{c})=2 \pi$ resp. $E(\widetilde{c})=2 \pi^{2}$ and $E\left(c_{s}\right)<2 \pi^{2}$ for $s \in(1 / 2,1]$. It follows that for sufficiently small $\epsilon>0$

$$
\left[f_{c}\right]=\left[f_{\widetilde{c}}\right] \in H_{n-1}\left(\Omega_{p} M, \Omega_{p}^{\leq 2 \pi^{2}-\epsilon} M ; \mathbb{Z}_{2}\right)
$$

and $c_{s}(t)=\widetilde{c}(t)$ for all $s \geq 1 / 2$ and all $t \in[0,1 / 2]$. Then we define a homotopy $\left(f_{\widetilde{c}}\right)_{s}$ of the mapping $f_{\widetilde{c}}: D^{n-1}(\rho) \longrightarrow \Omega_{p}^{\leq 2 \pi^{2} / 2} M$. At first we define a homotopy $\left(\bar{f}_{\widetilde{c}}\right)_{s}: D^{n-1}(\rho) \longrightarrow \Omega_{\bar{p}}^{\leq 2 \pi^{2}} M, s \in[0,1]$ of the mapping $\bar{f}_{\widetilde{c}}: D^{n-1} \longrightarrow \Omega_{\bar{p}}^{\leq 2 \pi^{2}} M:$

$$
\bar{f}_{\widetilde{c}, s}(v)(t)=\left\{\begin{array}{cc}
\bar{f}_{\widetilde{c}}(v)(t) & ; \quad 0 \leq t \leq 1 / 2 \\
c_{s}(1 / 2+2 t) & ; \quad 1 / 2 \leq t \leq 1
\end{array} .\right.
$$

Then $E\left(\bar{f}_{\widetilde{c}, s}(v)\right) \leq 2 \pi^{2}$ for all $s \in[0,1]$ and $E\left(\bar{f}_{\widetilde{c}, s}(v)\right)<2 \pi^{2}$ for all $v \in D^{n-1}(\rho), s>1 / 2$. We use the negative gradient flow $\Phi^{s}: \Omega_{p} M \longrightarrow$ $\Omega_{p} M, s \geq 0$ of the energy functional to define: $f_{c, s}(v):=\Phi^{1}\left(\bar{f}_{c, s}(v)\right)$. Then $E\left(f_{c, s}(v)\right) \leq 2 \pi^{2}$ for all $v \in D^{n-1}$ and $s \in[0,1]$ and $E\left(f_{c, s}(v)\right)<2 \pi^{2}$ for all $v \in D^{n-1}, s>1 / 2$. Hence the homotopy $f_{c, s}, s \in[0,1]$ shows $\operatorname{cr}\left(\left[f_{c}\right]\right)=$ $\operatorname{cr}\left(\left[f_{\widetilde{c}}\right]\right)<2 \pi$.

\section{Proof of Theorem 1}

(a) Lemma 2 shows that $\operatorname{crl}_{p}(M, g) \leq 2 \pi$ for all $p \in M$. Hence also $\operatorname{crl}(M, g) \leq 2 \pi$ by Equation (5).

(b) Let $h_{p} \in H_{k}\left(\Omega_{p} M, p ; \mathbb{Z}_{2}\right)$ with $\operatorname{crl}_{p}(M, g)=\operatorname{cr}(h)>\pi$. Lemma 2(b) implies $k=n-1$. Hence the manifold $M$ is $(n-1)$-connected and hence by 
the solution of the Poincaré conjecture homeomorphic to $S^{n}$. If $\mathrm{crl}(M, g)>\pi$ then $\operatorname{crl}_{p}(M, g)>\pi$ by Equation (5).

(c) If $\operatorname{crl}(M, g)=2 \pi$ Equation (5) and part (a) imply that $\operatorname{crl}_{p}(M, g)=2 \pi$ for all $p \in M$. Hence we assume now $\operatorname{crl}_{p}(M, g)=2 \pi$ for some $p \in M$. Part (b) implies that $M$ is homeomorphic to $S^{n}$ and $\operatorname{crl}_{p}(M, g)=\operatorname{cr}\left(h_{p}\right)$, where $h_{p} \in H_{n-1}\left(\Omega_{p q} M ; \mathbb{Z}_{2}\right) \cong \mathbb{Z}_{2}$ is non-zero. Choose a sequence $p_{j}$ of regular points of $\exp _{p}$ with $\lim _{j \rightarrow \infty} d\left(p, p_{j}\right)=0$, cf. for example [18, Cor. 18.2]. Then $E: \Omega_{p p_{j}} M \longrightarrow \mathbb{R}$ is a Morse function for any $j \geq 1$, cf. Section 3. This holds since $p_{j}$ as a regular point of $\exp _{p}$ is not conjugate to $p$ along any geodesic joining $p$ and $q$. Using the homotopy equivalence $\zeta_{p_{j} p}: \Omega_{p} M \longrightarrow \Omega_{p p_{j}} M$ introduced in Lemma 1 we define

$$
a_{j}:=\operatorname{cr}\left(\left(\zeta_{p_{j} p}\right)_{*}\left(h_{p}\right)\right),
$$

here $\left(\zeta_{p_{j} p}\right)_{*}\left(h_{p}\right) \in H_{n-1}\left(\Omega_{p p_{j}} M ; \mathbb{Z}_{2}\right)$.

Then Equation (7) implies that $\lim _{j \rightarrow \infty} a_{j}=2 \pi^{2}$ since $p=\lim _{j \rightarrow \infty} p_{j}$.

Morse theory implies that for any $j \geq 1$ there is a geodesic $c_{j} \in \Omega_{p p_{j}} M$ and $\epsilon_{j}>0$ with $a_{j}=E\left(c_{j}\right), \operatorname{ind}_{0}\left(c_{j}\right)=n-1$ such that the mapping

$$
f_{j}=f_{c_{j}}: D^{n-1}(\rho) \longrightarrow \Omega_{p p_{j}} M
$$

introduced in Definition 1 resp. Equation (9) satisfies: $a_{j}-\epsilon_{j}=$ $\max \left\{F\left(f_{j}(v)\right) ; v \in \partial D^{n-1}\right\}$, cf. Lemma 3. In addition we can assume that for any $\delta \in\left(0, \epsilon_{j}\right]$ the relative homology class

$$
0 \neq\left[f_{j}\right] \in H_{n-1}\left(\Omega_{p p_{j}} M, \Omega_{p p_{j}}^{\leq a_{j}-\delta} M ; \mathbb{Z}_{2}\right)
$$

is non-zero. This follows from Lemma 3 (b) and (c).

A subsequence $\left(c_{j}\right)_{j}$ converges to a geodesic loop $c \in \Omega_{p} M$, which we also denote by $\left(c_{j}\right)_{j}$. Then we obtain for the maps $f_{j}, j \in \mathbb{N}$ and the map $f_{c}: D^{n-1}(\rho) \longrightarrow \Omega_{p} M$ the following statements:

$$
\lim _{j \rightarrow \infty} \sup \left\{d\left(f_{j}(v)(t), f_{c}(v)(t)\right), t \in[0,1]\right\}=0
$$

and

$$
E\left(f_{c}(v)\right)=\lim _{j \rightarrow \infty} E\left(f_{j}(v)\right) .
$$

Since $\epsilon^{\prime}:=2 \pi-\max \left\{F\left(f_{c}(v)\right) ; v \in \partial D^{n-1}\right\}>0$ by Lemma 3 and $\epsilon^{\prime}=$ $\lim _{j \rightarrow \infty} \epsilon_{j}$ we obtain that $\epsilon_{j} \geq \epsilon^{\prime} / 2$ for all $j \geq j_{1}$ for some $j_{1} \in \mathbb{N}$.

Then

$$
\bar{f}_{\epsilon}=\left[f_{c}\right]=\left[\zeta_{p p_{j}} \circ f_{j}\right] \in H_{n-1}\left(\Omega_{p} M, \Omega_{p}^{\leq 2 \pi-\delta / 4} M\right) \neq 0
$$

for all $\delta \in\left(0, \epsilon^{\prime}\right]$. Equation (27) implies that $f_{c}$ and $\zeta_{p_{j} p} \circ f_{j}$ are homotopic for sufficiently large $j$, which implies the second equality of Equation (29). Since Equation (29) holds for any $\delta \in\left(0, \epsilon^{\prime}\right]$ we conclude for the critical value $\operatorname{cr}\left(\bar{f}_{\epsilon}\right)=2 \pi$. Lemma 5 implies that the Riemannian metric $g$ has constant sectional curvature. This finishes the proof of Theorem 1 . 


\section{Morse CONDition And CRitical LENGTH}

Instead of comparing the sectional curvature $K$ of the Riemannian metric $g$ with the sectional curvature 1 of the standard metric $g_{1}$ on $S^{n}$ we can compare the metrics $g$ and $g_{1}$ on $S^{n}$. For existence results of closed geodesics on spheres instead of the assumption $1 \leq K \leq 4$ several authors considered the assumption $g_{1} / 4<g<g_{1}$, the so-called Morse condition, cf. [3, Sec.3]. Instead of the lower bound $K \geq 1$ for the sectional curvature in Theorem 1 we use the assumption $g \leq g_{1}$ and obtain the following

Proposition 2. Let $g$ be a Riemannian metric on the $n$-sphere $S^{n}$ and $g_{1}$ be the standard Riemannian metric of constant sectional curvature 1 on $S^{n}$ such that $g \leq g_{1}$.

(a) For all $p \in M: \operatorname{crl}_{p}\left(S^{n}, g\right) \leq 2 \pi$ and $\operatorname{crl}\left(S^{n}, g\right) \leq 2 \pi$.

(b) If $\mathrm{crl}\left(S^{n}, g\right)=2 \pi$ then $g=g_{1}$.

Proof. (a) The assumption $g \leq g_{1}$ implies that for any $h \in H_{k}(X ; X \leq b)$ the critical value $\operatorname{cr}(h)$ with respect to the metric $g$ satisfies $\operatorname{cr}(h) \leq \operatorname{cr}_{1}(h)$, where $\operatorname{cr}_{1}(h)$ is the critical value with respect to the standard metric. Since $\operatorname{crl}_{p}\left(S^{n}, g\right)=\sqrt{2 \operatorname{cr}\left(h_{p}\right)}$ for a generator $h_{p} \in H_{n-1}\left(\Omega_{p} S^{n} ; \mathbb{Z}_{2}\right)$ resp. $\operatorname{crl}\left(S^{n}, g\right)=$ $\sqrt{2 \mathrm{cr}(h)}$ for a generator $h \in H_{n-1}\left(\Lambda S^{n}, \Lambda^{0} S^{n} ; \mathbb{Z}_{2}\right)$ the claim follows.

(b) Assume that for some $p \in S^{n}$ there is a tangent vector $w \in T_{-p} S^{n}$ with $g(w, w)<g_{1}(w, w)$.

Let $T_{p}^{2 \pi} S^{n}:=\left\{v \in T_{p} S^{n} ; g_{1}(v, v)=4 \pi^{2}\right\}$. Then we define a map $\Gamma: v \in$ $T_{p}^{2 \pi} S^{n} \cong S^{n-1} \longmapsto \Gamma(v) \in \Omega_{p} S^{n}$ as follows: Let $\gamma_{v}:[0,1] \longrightarrow S^{n}$ be the great circle parametrized proportional to arc length with $p=\gamma_{v}(0)=\gamma_{v}(1)$ and $v=\gamma_{v}^{\prime}(0)$. In particular $\gamma_{v}(1 / 2)=-\gamma_{v}(0)=-p$. Let $w_{1} \in T_{p} S^{n}$ be the vector such that $w=\gamma_{w_{1}}^{\prime}(1 / 2)$. We define

$$
\Gamma(v)(t)=\left\{\begin{array}{ccc}
\gamma_{v}(t) & ; \quad 0 \leq t \leq 1 / 2 \\
\gamma_{w_{1}}(t) & ; & 1 / 2 \leq t \leq 1
\end{array} .\right.
$$

Then the map $(t, v) \in S^{1} \times S^{n-1} \longmapsto \Gamma(v)(t) \in S^{n}$ defines a homotopically non-trivial map of degree 1 . Hence $\Gamma$ represents the non-trivial class $h_{p}$. Choose the negative gradient flow $\Phi^{s}: \Omega_{p} S^{n} \longrightarrow \Omega_{p} S^{n}$ of the energy functional $E$ with respect to the metric $g$. Since $E\left(\Gamma\left(w_{1}\right)\right)=E\left(\gamma_{w_{1}}\right)<2 \pi^{2}$ by assumption and since $\Gamma(v)$ is not smooth for any $v \neq w_{1}$ we obtain $E\left(\Phi^{1} \circ\right.$ $\Gamma(v))<2 \pi^{2}$ for all $v \in T_{p}^{2 \pi} S^{n}$. Therefore $\operatorname{crl}_{p}\left(S^{n}, g\right)=\sqrt{2 \operatorname{cr}\left(\left[\Phi^{1} \circ \Gamma\right]\right)}<$ $2 \pi$.

For a compact Riemannian manifold $(M, g)$ and a point $p \in M$ define $d_{p}=\sup \{d(p . q) ; q \in M\}$. Then the diameter diam is given as diam $=$ $\sup \left\{d_{p} ; p \in M\right\}$ and the radius by $\operatorname{rad}=\inf \left\{d_{p} ; p \in M\right\}$.

Proposition 3. For a compact Riemannian manifold $(M, g)$ the critical length $\mathrm{Crl}_{p}(M, g)$ at the point $p$ satisfies $\mathrm{crl}_{p}(M, g) \geq 2 d_{p}$. In particular we obtain $\inf \left\{\operatorname{crl}_{p}(M, g) ; p \in M\right\} \geq 2 \operatorname{rad}(M, g)$.

Proof. Let $h_{p} \in H_{n-1}\left(\Omega_{p} S^{n} ; \mathbb{Z}_{2}\right)$ be the generator, hence $\mathrm{crl}_{p}=\sqrt{2 \mathrm{cr}\left(h_{p}\right)}$. Choose a map $\xi_{p}: S^{n-1} \longrightarrow \Omega_{p} S^{n}$ representing $h_{p}$. Then the map $(t, x) \in$ $[0,1] /\{0,1\} \times S^{n-1} \longmapsto \xi_{p}(x)(t) \in S^{n}$ is a map of degree 1 , in particular the map is surjective. Hence $\sup \left\{L\left(\xi_{p}(x)\right) ; x \in S^{n-1}\right\} \geq 2 d_{p}$. 
Example 1. One can write the standard metric $g_{1}$ on $S^{2}$ as warped product $d t^{2}+\sin ^{2} t d x^{2}$ in the coordinates $(t, x) \in[0, \pi] \times[0,2 \pi] /\{0,2 \pi\}=[0, \pi] \times$ $\mathbb{Z} /(2 \pi \mathbb{Z})$. Denote by $p=(0, x)$ resp. $p^{\prime}=(\pi, x)$ the coordinate singularities. Choose a one-parameter family of smooth functions $f_{r}:[-\pi, \pi] \longrightarrow \mathbb{R}, r \in$ $(0,1]$ with $f_{r}^{\prime}(0)=1, f_{r}(\pi / 2)=r$ and $f_{1}(t)=\sin t, f_{r}(t)=-f_{r}(-t), 0<$ $f_{r}(t) \leq \sin t, f_{r}(\pi / 2+t)=f_{r}(\pi / 2-t)$ and $f_{r}^{\prime \prime}(t)<0$ for all $t \in(0, \pi)$.

Then the warped product metric $d t^{2}+f_{r}^{2}(t) d x^{2}$ on $(0, \pi) \times(0,2 \pi) /\{0,2 \pi\}$ extends smoothly to a Riemannian metric $g=g_{r}$ on $S^{2}$.

The Gauss curvature $K(t, x)=-f_{r}^{\prime \prime}(t) / f_{r}(t)$ is positive everywhere. The surfaces can be seen as surfaces of revolution generated by a one-parameter family of convex curves $t \in[0,2 \pi] \longmapsto c_{r}(t)=(x(t), z(t)) \in[0, r] \times[-\pi, \pi]$ parametrized by arc length, which intersect at $t=0$ and $t=\pi$ the axis of revolution orthogonally, having distance $\leq r$ from the axis of revolution and such that $c_{1}$ is a half-circle.

For $r<1$ we have $g \leq g_{1}$ and $g \neq g_{1}$. Since all geodesics starting from the point $p$ are closed and have length $2 \pi$ we obtain $\operatorname{crl}_{p}\left(S^{n}, g\right)=2 \pi$. For any other point $q \in S^{2}$ the distance to $p$ or to $p^{\prime}$ is at least $\pi / 2$. Therefore $\operatorname{crl}_{q}\left(S^{2}, g_{r}\right) \geq \pi$. For $t \in[0, \pi]$ the curve $s \in[0,1] \longmapsto \gamma_{t}(s)=(t, 2 \pi s)$ is a circle of length $2 \pi f(t)$ on the surface of revolution. Hence the map $t \in([0, \pi],\{0, \pi\}) \longmapsto \gamma_{t} \in\left(\Lambda S^{2}, \Lambda^{0} S^{2}\right)$ represents the generator $h \in$ $H_{1}\left(\Lambda S^{2}, \Lambda^{0} ; \mathbb{Z}_{2}\right)$. Therefore $\operatorname{crl}\left(S^{n}, g\right) \leq 2 \pi f(\pi / 2)=2 \pi r$. Hence we obtain a one-parameter family $g_{r}, r \in(0,1)$ of convex surfaces $\left(S^{2}, g_{r}\right)$ of revolution with $\operatorname{cr}_{q}\left(S^{2}, g_{r}\right) \geq \pi$ for all $q, \operatorname{cr}_{p}\left(S^{2}, g_{r}\right)=\operatorname{cr}_{p^{\prime}}\left(S^{2}, g_{r}\right)=2 \pi, g \leq g_{1}$ and $\lim _{r \rightarrow 0} \operatorname{cr}\left(S^{2}, g_{r}\right)=0$. This example shows that in Part (b) of Proposition 2 it is not sufficient to assume that $\mathrm{crl}_{p}(M, g)=2 \pi$.

\section{REFERENCES}

[1] F. Balacheff, C. Croke, and M. Katz. A Zoll counterexample to a geodesic length conjecture. Geom. Funct. Anal., 19(1):1-10, 2009.

[2] W. Ballmann. Über Geschlossene Geodätische, Habilitationsschrift Math. Naturwiss. Fak. Univ. Bonn. 1983.

[3] W. Ballmann, G. Thorbergsson, and W. Ziller. Existence of closed geodesics on positively curved manifolds. J. Differential Geom., 18(2):221-252, 1983.

[4] W. Ballmann, G. Thorbergsson, and W. Ziller. Some existence theorems for closed geodesics. Comment. Math. Helv., 58(3):416-432, 1983.

[5] G. D. Birkhoff. Dynamical systems with two degrees of freedom. Trans. Amer. Math. Soc., 18(2):199-300, 1917.

[6] R. Bott and H. Samelson. Applications of the theory of Morse to symmetric spaces. Amer. J. Math., 80:964-1029, 1958.

[7] J. Cheeger and D. Ebin. Comparison theorems in Riemannian geometry, volume 9 of Math. Libr. North-Holland Publ. Comp.,, Amsterdam, Oxford, 1975, Reprint Amer. Math. Soc. 2008.

[8] J.-H. Eschenburg. Comparison theorems and hypersurfaces. Manuscripta Math., 59(3):295-323, 1987.

[9] K. Grove and K. Shiohama. A generalized sphere theorem. Ann. of Math. (2), 106(2):201-211, 1977.

[10] N. Hingston. Equivariant Morse theory and closed geodesics. J. Differential Geom., 19(1):85-116, 1984.

[11] N. Hingston and H.-B. Rademacher. Resonance for loop homology of spheres. J. Differential Geom., 93(1):133-174, 2013. 
[12] Y. Itokawa and R. Kobayashi. The length of the shortest closed geodesics on a positively curved manifold. Max-Planck-Institute for Mathematics Bonn, Preprint 199143, 1991.

[13] Y. Itokawa and R. Kobayashi. The length of the shortest closed geodesics on a positively curved manifold. arXiv:math/0507489, July 2005.

[14] Y. Itokawa and R. Kobayashi. The length of the shortest closed geodesics on a positively curved manifold. arXiv:math/0805.2793, May 2008.

[15] W. Klingenberg. Lectures on closed geodesics. Springer-Verlag, Berlin-New York, 1978. Grundlehren der Mathematischen Wissenschaften, Vol. 230.

[16] W. Klingenberg. Riemannian geometry, volume 1 of De Gruyter Studies in Mathematics. Walter de Gruyter \& Co., Berlin, second edition, 1995.

[17] L. A. Lyusternik and A. I. Fet. Variational problems on closed manifolds. Doklady Akad. Nauk SSSR (N.S.), 81:17-18, 1951 (Russian).

[18] J. Milnor. Morse theory. Based on lecture notes by M. Spivak and R. Wells. Annals of Mathematics Studies, No. 51. Princeton University Press, Princeton, N.J., 1963.

[19] M. Sugimoto. On Riemannian manifolds with a certain closed geodesic. Tôhoku Math. J. (2), 22:56-64, 1970.

[20] V. A. Toponogov. Riemannian spaces having their curvature bounded below by a positive number. Dokl. Akad. Nauk SSSR, 120:719-721, 1958 (Russian).

[21] V. A. Toponogov. Evaluation of the length of a closed geodesic on a convex surface. Dokl. Akad. Nauk SSSR, 124:282-284, 1959 (Russian).

Mathematisches Institut, Universität Leipzig, 04081 Leipzig, Germany

E-mail address: rademacher@math.uni-leipzig.de 